\title{
ENERGY-EFFICIENT POWER ALLOCATION FOR IMPERFECT CSI DOWNLINK NOMA SYSTEM
}

\author{
Reem Aldebes $^{1}$, Kaharudin Dimyati ${ }^{1}$, and Effariza Hanafi ${ }^{1}$ \\ ${ }^{1}$ Department of Electrical Engineering, Faculty of Engineering, University of Malaya, Kuala Lumpur, \\ Malaysia, Tel: +60134468093, e-mail: effarizahanafi@um.edu.my
}

Received Date: March 26, 2020; Revised Date: November 20, 2020; Acceptance Date: February 9, 2021

\begin{abstract}
The fifth generation $(5 \mathrm{G})$ networks must provide the massively increased number of users by thousand times higher data rate at lower power consumption. Thus, optimizing the energy efficiency (EE) becomes an essential issue that has to be researched from the green communication perspective. Non-orthogonal multiple access (NOMA) is considered one of the high potential techniques in fifth-generation systems. This technology is favorable to maximize the energy efficiency and the spectrum efficiency by composing different signals at the same time on the same carrier at different power levels. In this paper, a low complexity power allocation algorithm is proposed in imperfect channel state information (CSI) downlink NOMA cellular system, where obtaining full CSI at the base station is considered a challenge. The proposed algorithm relies on the fact that the allocated power is inversely proportional to the channel strength of the user to implement the successive interference cancellation (SIC) technique at the user terminal to reconstruct the desired signal. The performance of the system is analyzed in terms of energy efficiency and outage probability and compared to the conventional orthogonal multiple access (OMA) system. Results show that the proposed algorithm increases the energy efficiency by about 50\% compared to the conventional OMA technology, and an improvement in the outage probability has been achieved. Furthermore, the effect of the error in the channel estimation on the energy efficiency in imperfect CSI NOMA system is evaluated. The simulation shows that the energy efficiency reduces when the channel estimation error increases; and the best performance is achieved in the perfect CSI case where the channel estimation error is zero.
\end{abstract}

Keywords: Downlink cellular system, Energy efficiency, Imperfect channel state information, Outage probability

\section{Introduction}

The numbers of connected equipment will massively increase in the fifth generation $(5 \mathrm{G})$ compared to the previous $4 \mathrm{G}$ networks $[1,2]$. By 2030, the density of the connected devices is expected to reach $10^{7}$ devices $/ \mathrm{km}^{2}$, and multimedia applications will be the most popular applications for the users, such as mobile video calls, streaming videos, and online games. As a result, the required data rate will raise about 10 times more than that in $4 \mathrm{G}$, and the peak transmitted data in the $5 \mathrm{G}$ is expected to be about $20 \mathrm{Gbps}[3,4]$. On the other hand, the spectrum efficiency and the energy efficiency should be degraded in 5G by $\mathrm{x} 5$ and $\mathrm{x} 100$ times, respectively [4]. Thereby, satisfying the requirements of a massive number of users within the network limited resources is considered a challenge in $5 \mathrm{G}$.

Implementing orthogonal multiple access (OMA) algorithms in 5G will not be adequate due to the limited number of simultaneously transmitted signals within the orthogonal resources [5]. On the other hand, non-orthogonal multiple access (NOMA) is considered a high potential technique to provide the increased number of users in $5 \mathrm{G}$ by 
the required quality of service. In the NOMA system, each carrier frequency is utilized to transmit superposed signals of multiple users at the same time within the same cell. Thus, the NOMA technology improved spectral efficiency (SE), energy efficiency (EE), and the total throughput. The available NOMA techniques can broadly be divided into two categories, namely, power-domain and code-domain NOMA [6]. The base station (BS) in the power-domain NOMA system allocates more power to the weaker channel to increase the overall throughput. With this strategy, the strong channel user can eliminate the interference due to the weaker channel by using the successive interference cancellation (SIC) techniques, and thus the system fairness is accomplished [6]. This paper focuses on the power-domain NOMA system, where different power levels are allocated to the users in the cell to attain the optimization of energy efficiency.

Power allocation (PA) is considered an essential method to raise the data-rate and the energy efficiency in the NOMA system, where various powers are assigned to the cell's users and combined on the same subcarrier at the same time [7]. A game-theoretic power allocation mechanism is studied in [8] where power is assigned to the users to maximize the total throughput and the average throughput in the downlink NOMA cellular system. However, the investigation of the system performance based on the proposed algorithm in [8] is under the assumption that the BS has perfect channel state information (CSI) which is difficult to achieve. Perfect CSI is not easy to achieve because of channel estimation errors, feedback, and quantization errors. It is requisite to search novel solutions that address the imperfect CSI in wireless communication systems [9].

Moreover, energy efficiency receives significant attention from both academia and industry researches since the information and communication sector consumes $5 \%$ of the total global energy consumption [10]. Hence, many researchers have concentrated on energy efficiency in the NOMA system [11-13]. An energy-efficient novel power allocation algorithm is presented in [9] where the optimization problem is formulated based on imperfect CSI with outage probability constraints and then it is relaxed to a non-probabilistic problem. The results obtained of a small cell of one BS and 2 users showed that the performance of the proposed algorithm is better than the conventional algorithms.

Optimizing the energy efficiency in the single input single output NOMA system (SISO) NOMA system has been studied in [10] where the proposed power allocation algorithm for 2 users in a single cell shows superior behavior compared to the traditional algorithms. However, more investigation is still required about this algorithm in the imperfect CSI case. Distance between the receiver and the BS has been used for power allocation in $[10,14]$. On the other hand, the joint optimization algorithm has been proposed in [15] where the power allocation utilizes to minimize the transmitted power which is required to attain the minimum required rate. Optimizing energy efficiency for imperfect CSI case with two secondary users is studied in [16]. The authors in [17] studied energy efficiency optimization and proved that NOMA outperforms conventional OMA systems under low user's quality of service constraint.

In this paper, a simple PA algorithm among $\mathrm{M}$ users is proposed for an imperfect CSI downlink NOMA system where the allocated power level to each channel in the cell is determined based on the channel strength. The proposed algorithm performance in a single cell is investigated in terms of energy efficiency and outage probability, and it is compared with the conventional OMA. The rest of this paper is arranged as follows: Section II illustrates the cellular downlink NOMA system model and the proposed power allocation mechanism. The numerical results are illustrated and discussed in Section III. Finally, a conclusion is presented in Section IV. 


\section{System Model and PA Algorithm}

\section{System Model}

The resources of a single cell downlink NOMA system are shared among $M$ users' equipment (UEs) to provide them with the required service. Here, the transmitter and each UE are provided by a single antenna [18], and superposition coding techniques are used to provide all receivers by the service at the same time. The received signal at $\mathrm{UE}_{\mathrm{m}}$ is defined as [16]

$$
y_{m}(t)=\left(h_{m}+e_{h}\right) x(t)+n_{m}, \quad \forall m \in\{1,2, \ldots, M\}
$$

where $h_{m}$ is the BS-UE $E_{m}$ channel gain, $e_{h}$ is the channel estimation error, $n_{m}$ is a standard complex normal random variable $\sim C N\left(0, \sigma^{2}\right)$ which represents the additive white Gaussian noise (AWGN) at $\mathrm{UE}_{\mathrm{m}}$ with zero mean and variance $\sigma^{2}$, and $x(t)$ represents the transmitted signal which is given as [8]

$$
x(t)=\sum_{m=1}^{M} \sqrt{\alpha_{m} P_{t}} x_{m}(t),
$$

where $x_{m}(t)$ is the individual orthogonal frequency division multiplexed (OFDM) signal, $P_{t}$ is the total BS's transmitted power and $\alpha_{m}$ is the coefficient of the $m$-th user's power, which verifies:

$$
\sum_{m=1}^{M} \alpha_{m}=1 .
$$

$M$ different signals are combined on the same carrier, transmitted by the BS, and then, are received by all users [19]. These users have been ordered depending on their channels' strength such that $\left|h_{M}\right| \geq\left|h_{M-1}\right| \geq \ldots \geq\left|h_{2}\right| \geq\left|h_{1}\right|$. The closest user to BS has the strongest channel $h_{M}$, and it is denoted as $\mathrm{UE}_{\mathrm{M}}$, while the farther user, which has the weakest channel $h_{1}$, is denoted as $\mathrm{UE}_{1}$. The SIC technique could be utilized to extract a specific signal from superposed signals on a single carrier. Thus, a higher-order user can decode the signals of the lower order users then it decodes its signal. Time-division multiple access (TDMA) is considered a conventional OMA system for the comparison purpose with the NOMA system $[17,20,21]$. The total BS power is utilized to transmit each signal in the TDMA within a time slot $T_{\mathrm{i}}$ [20]. For a fair comparison with the NOMA, the total transmission time in the NOMA should be equal the sum of the time slots in the OMA $\left(\sum_{i=1}^{M} T_{i}=1\right)$ [20]. Both of the NOMA system model and the conventional OMA system model are illustrated in Figure 1.

Practically, it is a challenge to obtain perfect CSI. Thus, an error in the channel estimation is considered as shown in Equation (1). To enable the NOMA users to perform SIC and to detect the signals of the lower order users, the BS allocates the power levels according to their channel gains. That is, each receiver will eliminate the signals of other combined users on the same carrier which have weaker channel gain. On the other hand, the signals of the higher order users with stronger channel gains will represent inter-interference signals. To maximize the data rate, the effect of this interference should be minimized, and that could be ensured when the allocated power to the stronger channel user is lower than the allocated power to the weaker channel user $\left(P_{m} \geq P_{m+1}\right)$ [22]. On the other side, the BS in the OMA system allocates the total transmission power to $\mathrm{m}$-th user during the $\mathrm{m}$-th time slot. Thus, signal to interference and noise ratio $\left(\right.$ SINR) at $\mathrm{UE}_{\mathrm{m}}$ in both systems can be expressed as $[16,23]$ 


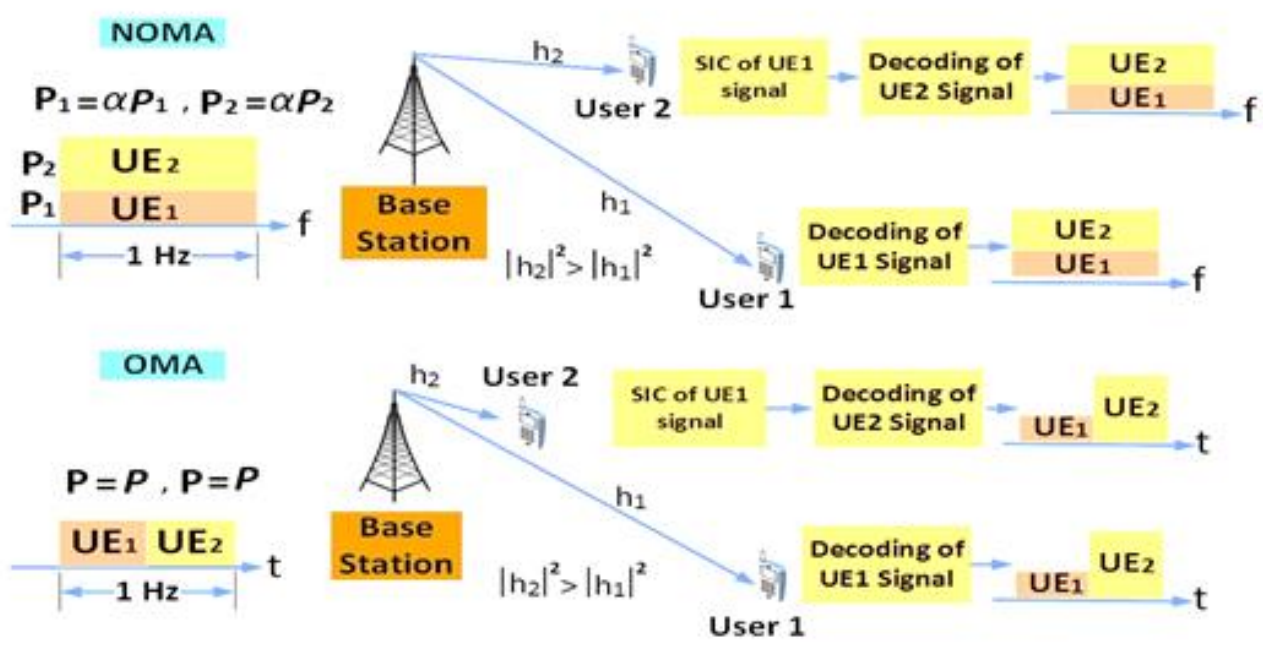

Figure 1. A comparison between the OMA and the NOMA system models

$$
\begin{gathered}
\operatorname{SINR}_{m}{ }^{\text {NOMA }}=\frac{P_{m}\left|h_{m}\right|^{2}}{\left|h_{m}\right|^{2} \sum_{i=m+1}^{M} P_{i}+\sigma_{e}^{2} \sum_{i=1}^{M} P_{i}+\sigma^{2}}, \\
\operatorname{SINR}_{m}{ }^{O M A}=\frac{P\left|h_{m}\right|^{2}}{\sigma_{e}^{2} P+\sigma^{2}},
\end{gathered}
$$

where $P_{m}$ represents the allocated power to $\mathrm{UE}_{\mathrm{m}}, \sum_{i=m+1}^{M} P_{i}$ represents undesired signals from the higher-order users, and $\sigma_{e}{ }^{2}$ represents the power fraction due to the channel estimation error. $P$ is the total transmission power of the BS which is equal for all users but for a specific time. Thus, the data rate of $\mathrm{UE}_{\mathrm{m}}$ is defined as $[17,24]$

$$
\begin{aligned}
R_{m}{ }^{\text {NOMA }}=\log _{2}\left(1+\frac{P_{m}\left|h_{m}\right|^{2}}{\left|h_{m}\right|^{2} \sum_{i=m+1}^{M} P_{i}+\sigma_{e} \sum_{i=1}^{M} P_{i}+\sigma^{2}}\right), \\
R_{m}{ }^{\text {OMA }}=\frac{1}{M} \log _{2}\left(1+\frac{P\left|h_{m}\right|^{2}}{\sigma_{e}{ }^{2} P+\sigma^{2}}\right) .
\end{aligned}
$$

It is obvious that there is no interference from the other users on the signal of the $\mathrm{m}$-th user during its transmission time and this causes an increase in the $\operatorname{SINR}_{m}$ in the OMA system. On the other hand, that data rate decreases as the number of users increases in the OMA system because of the bandwidth sharing [24]. The total data rate, $R_{\text {sum }}$ of a cell of a single BS that serves $M$ users is expressed as

$$
R_{\text {sum }}=\sum_{m=1}^{M} R_{m}
$$


The system's energy efficiency is defined as the ratio of total data rate to total consumption power, which is expressed as [9]

$$
\begin{gathered}
E E=\frac{R_{\text {sum }}}{P_{t}+P_{c}} \\
E E^{\text {NOMA }}=\frac{\sum_{m=1}^{M} \log _{2}\left[1+\frac{P_{m}\left|h_{m}\right|^{2}}{\left|h_{m}\right|^{2} \sum_{i=m+1}^{M} P_{i}+\sigma_{e}^{2} \sum_{i=1}^{M} P_{i}+\sigma^{2}}\right]}{\sum_{i=1}^{M} P_{i}+P_{c}}, \\
E E^{O M A}=\frac{\sum_{m=1}^{M} \frac{1}{M} \log _{2}\left[1+\frac{P\left|h_{m}\right|^{2}}{\sigma_{e}^{2} P+\sigma^{2}}\right]}{P+P_{c}},
\end{gathered}
$$

where $P_{c}$ is the BS's dissipated power. From Equation (7), the impact of the channel estimation error on the system EE is obvious in the imperfect CSI system. As the channel estimation error increases, the individual data rate and the total data rate will decrease. Therefore, at the same transmission power of the BS, the energy efficiency will degrade because of the decrease in the overall throughput. Moreover, the error of the channel estimation affects the energy efficiency in the OMA system more than the NOMA system, since this error is considered as the main part of the noise in the SINR formula. The best performance could be attained in the perfect CSI case when the channel estimation error is zero $\left(\sigma_{e}{ }^{2}=0\right)$ where the noise and interference power is minimized. This leads to the assumption that the perfect CSI NOMA case will show better performance

Here, our goal is to maximize the system's energy efficiency under a restriction of limited total consumption power. To implement SIC for extracting the desired signals at the receivers, the allocated power to the users should be ordered according to their channels' strength [19]. Hence, the problem could be formulated as

$$
\begin{gathered}
\text { Maximize } \quad E E=\frac{R_{\text {sum }}}{P_{t}+P_{c}} \\
\text { Subject to } \sum_{i=1}^{M} P_{i} \leq P_{t} \\
\forall P_{i} \geq 0 \\
P_{m} \geq P_{m+1}
\end{gathered}
$$

It is obvious from Equation (8) that maximizing energy efficiency requires maximizing the sum rate at a certain power consumption level.

\section{Power Allocation Algorithm}

In the downlink NOMA system, a higher power level should be set to the weaker user and a lower power level will be allocated to the strongest user. Based on this fact, a PA algorithm is proposed for the NOMA system in the imperfect CSI case. For simplicity, the variance of the channel estimation error is assumed to be equal for all users in the cell [17]. 
The allocated power $P_{m}$ to the m-th user is inversely proportional to the channel strength as follows

$$
P_{m}=\frac{\beta P_{t}}{\left|h_{m}\right|^{2}+\sigma_{h}^{2}},
$$

where $\frac{\beta}{\left|h_{m}\right|^{2}+\sigma_{h}^{2}}=\alpha_{m}$. By substituting it in Equation (3), $\beta$ can be expressed as

$$
\begin{gathered}
\sum_{m=1}^{M} \frac{\beta}{\left|h_{m}\right|^{2}+\sigma_{h}^{2}}=1 \\
\beta=\left[\sum_{i=1}^{M} \frac{1}{\left|h_{i}\right|^{2}+\sigma_{h}^{2}}\right]^{-1} .
\end{gathered}
$$

By substituting Equation (10) in Equation (9), $P_{m}$ can be re-written as

$$
P_{m}=\frac{P_{t}}{\left(\left|h_{m}\right|^{2}+\sigma_{h}^{2}\right)\left[\sum_{i=1}^{M} \frac{1}{\left|h_{i}\right|^{2}+\sigma_{h}^{2}}\right]} .
$$

From Equation (11), the allocated power to a user will decrease when the number of users rises. This will affect the SINR of the user at the cell edge and consequently, the coverage of the BS will degrade. Under constrained SINR level, the number of composed users on a single carrier in the NOMA system will be limited to guarantee the user's required QoS. However, these users still are provided by service at different carriers.

According to the proposed algorithm, when the SINR at the cell edge user is less than the threshold level, that user will be considered out of coverage. In this case, $\beta$ will be re-estimated based on the updated information, and the allocated power to other users will be re-evaluated. The proposed algorithm has been summarized in Algorithm 1 .

Algorithm 1. The Proposed Power Allocation Algorithm for Imperfect CSI NOMA System

1: Initiate $l=M$,

2. Determine $\beta$ based on Equation (10),

3. For $m=1: l$ do:

3.1 Evaluate the allocated power $P_{m}$ based on Equation (11),

3.2 Evaluate $\operatorname{SINR}_{m}$ based on Equation (4)

4. If $S I N R_{m}<$ threshold level

$4.1 l=l-1$

4.2 Repeat steps 2 to 4

5: End 


\section{Outage Probability}

Providing each user with the required quality of service is the aim of the downlink NOMA system. Thus the outage probability of the user at the edge of the cell is considered an important performance metric to evaluate the service. The definition of the outage probability $P_{r}$ at the m-th user, $P r_{m}{ }^{\text {out }}$, is the probability that the signal to interference and noise ratio of the user is less than a threshold value $\delta_{\text {th }}$ that represents the minimum required level of service [16]. For the imperfect CSI NOMA system, the outage probability could be expressed as

$$
\begin{gathered}
\operatorname{Pr}_{m}^{\text {out }}=\operatorname{Pr}\left[\operatorname{SINR}_{m} \leq \delta_{t h}\right] \\
=\operatorname{Pr}\left[\frac{\left|h_{m}\right|^{2} P_{m}}{\left|h_{m}\right|^{2} \sum_{i=m+1}^{M} P_{i}+\sigma_{\varepsilon}{ }^{2} \sum_{i=1}^{M} P_{i}+\sigma^{2}} \leq \delta_{t h}\right] \\
=\operatorname{Pr}\left[\left|h_{m}\right|^{2} \leq \tilde{\delta}_{m}\right]
\end{gathered}
$$

where $\tilde{\delta}_{m}$ is

$$
\tilde{\delta}_{m}=\delta_{t h} \frac{\sigma_{\varepsilon}{ }^{2} \sum_{i=1}^{M} P_{i}+\sigma^{2}}{P_{m}-\delta_{\text {th }} \sum_{i=m+1}^{M} P_{i}}
$$

That estimated value of the channel gain $\left|h_{m}\right|^{2}$ has an exponential distribution with unity mean and unity variance [16]. Thus, the outage probability of the $\mathrm{m}$-th user in the proposed imperfect CSI NOMA system is expressed as

$$
\operatorname{Pr}_{m}^{\text {out }}=\operatorname{Pr}\left[\left|h_{m}\right|^{2} \leq \tilde{\delta}_{m}\right]=\left\{\begin{array}{cc}
1-e^{-\widetilde{\delta}_{m}}, & \tilde{\delta}_{m} \geq 0 \\
0, & \tilde{\delta}_{m}<0
\end{array}\right.
$$

From Equation (14), it is seen that the outage probability increases when the required quality of service, $\delta_{t h}$ increases. Moreover, the increased number of users in the cell causes an increase in the term $\sum_{i=m+1}^{M} P_{i}$ for the user at the cell-edge, and therefore the outage probability will increase, too.

\section{Results and Discussion}

The proposed PA algorithm is evaluated in a single cellular cell with central BS, which serves $\mathrm{M}$ users, and the achieved results are compared to the conventional OMA in a zerochannel estimation error case. The users are randomly distributed within a single cell, where the BS-user channel has been modeled as $h_{m} \sim C N\left(0, \delta_{m}^{2}\right)$. For simplicity, and without loss the generality, the case of $M=3$ users and the variance of the channel gains are given by $\delta_{m}^{2}=\frac{m}{M}$ has been considered in this paper. The total transmission power $P_{t}$ is varied up to $1 \mathrm{~W}$ and the assumed total BS dissipated power is $P_{c}=1 \mathrm{~W}$. The system bandwidth and the AWGN spectral density are assumed to be $1 \mathrm{MHz}$ and $-174 \mathrm{dBm} / \mathrm{Hz}$ respectively [14]. Results are taken as an average of 1000 run trials. 


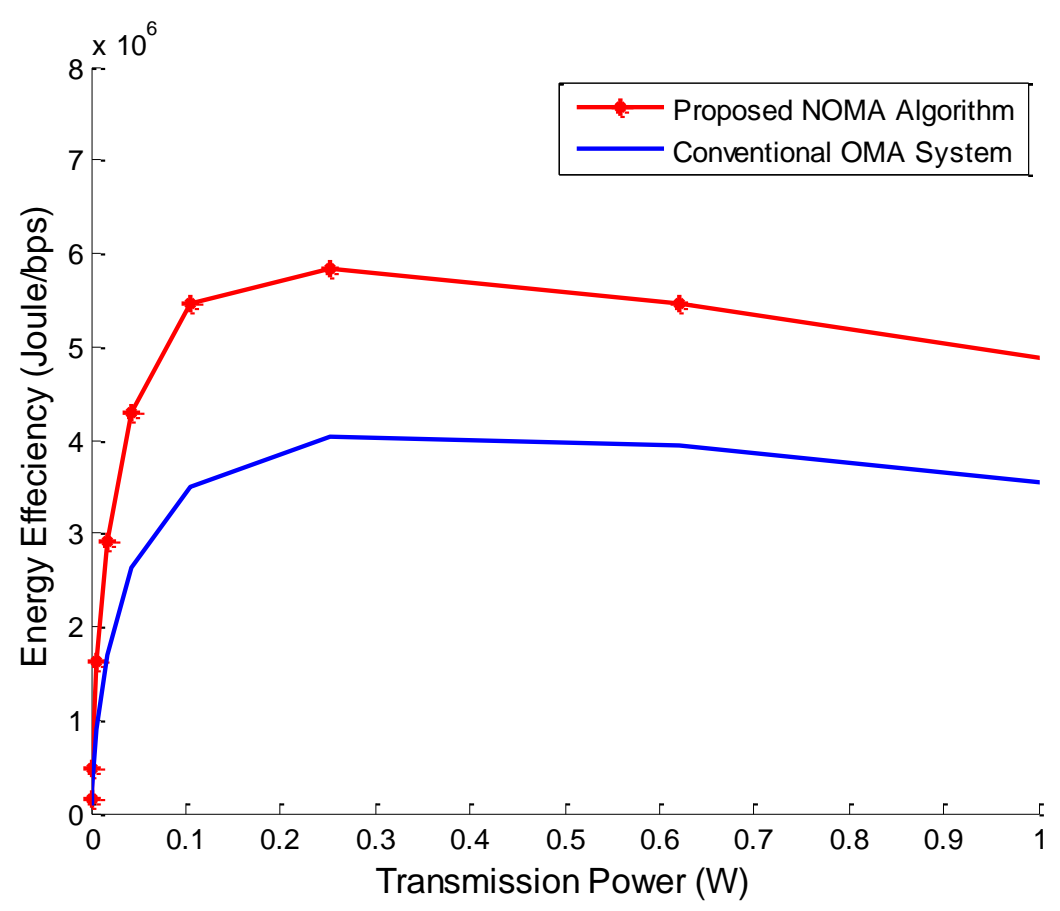

Figure 2. Energy efficiency versus transmission power

Figure 2 presents the obtained energy efficiency at different transmission powers. It illustrates that the proposed algorithm causes an EE enhancement compared with conventional OMA where the total transmission power is distributed equally among all users. For example, at $P_{t}=0.25 \mathrm{~W}$, the proposed algorithm achieves a more than $50 \%$ increase in energy efficiency compared to that in the OMA system. Moreover, the energy efficiency obtained by applying the proposed algorithm will increase when the transmission power increases until it reaches its maximum value at a certain $P_{t}$. Thus, any redundant power will not cause any increase in the energy efficiency.

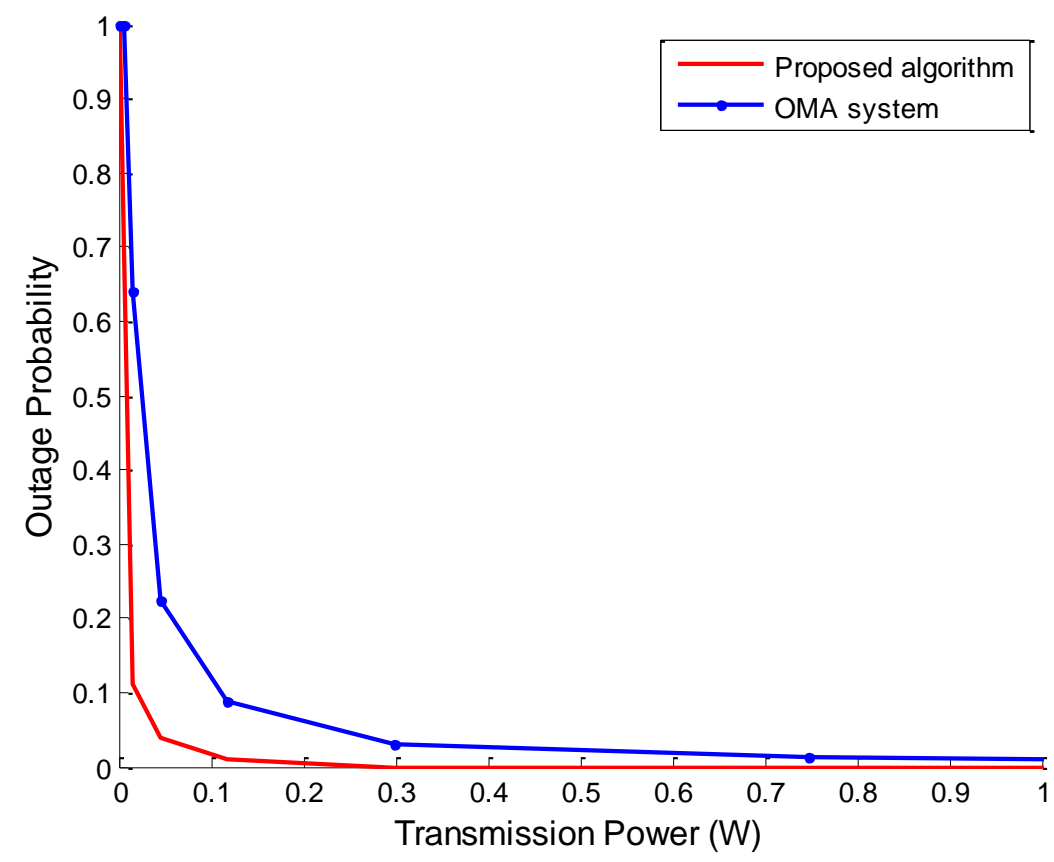

Figure 3. Outage probability versus transmission power 
The performance of the proposed algorithm is investigated in terms of the outage probability, too. Under the same circumstances in the previous experiments, and with threshold SINR=1 (minimum data rate is $1 \mathrm{bps}$ ), the outage probability of the cell-edge user is evaluated and presented in Figure 3. As the transmission power increases in both: NOMA and OMA systems, the outage probability decreases. Thus, the increase in the system resources will lead to better coverage and to enhance the quality of service of the user at the cell edge. The results illustrate that the proposed algorithm outperforms the conventional OMA in terms of outage probability. This indicates that for the same number of users and system resources, the coverage of the cell edge user is improved due to the proposed PA algorithm in the NOMA algorithm compared to the conventional OMA. When the number of users increases, the performance is expected to be worse than in the perfect CSI case.

In the second scenario, the proposed algorithm's performance is investigated in the imperfect CSI case. The error of channel estimation is varied while the other parameters remain the same. Figure 4 shows the energy efficiency for both the proposed NOMA algorithm and conventional OMA for different estimation error values. Figure 4 shows that in the studied cases, the proposed algorithm outperforms the conventional OMA in terms of energy efficiency. Moreover, results illustrate that both the algorithm's performance degrades when the estimation error increases, and the best performance is when the estimation error is zero (perfect CSI case) due to the higher SINR in this case.

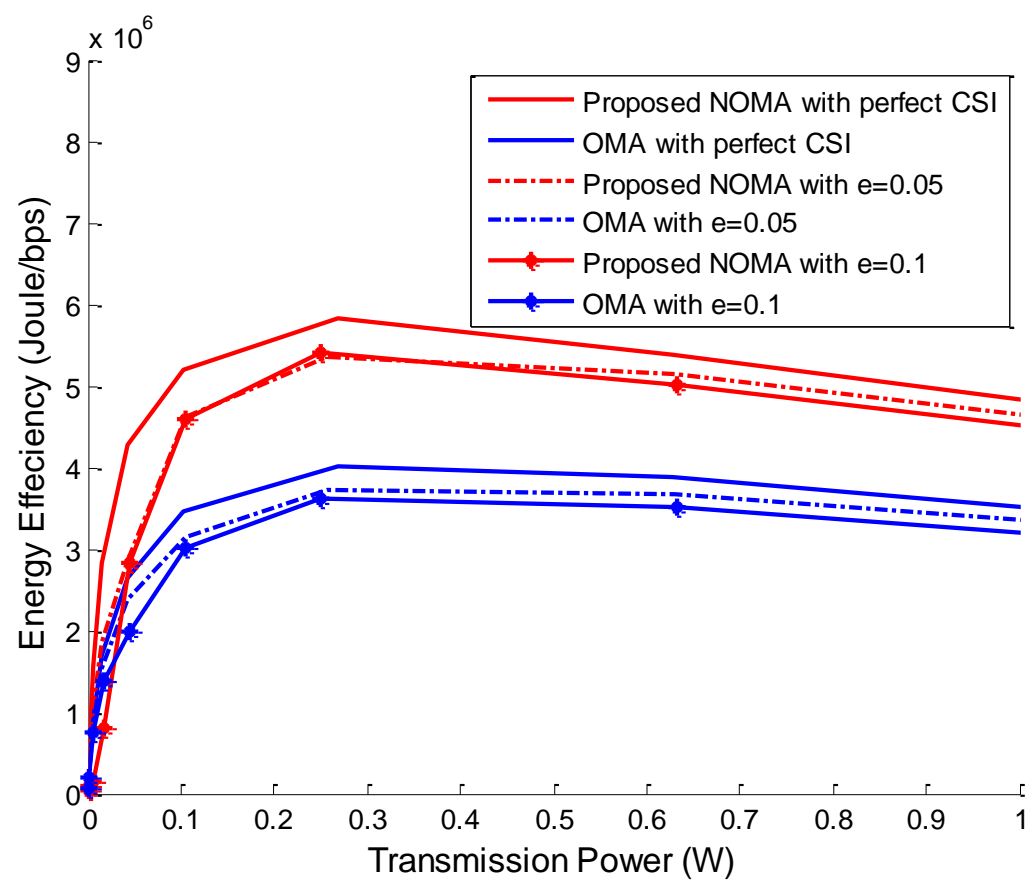

Figure 4. Energy efficiency versus transmission power at various estimation error values

In the next scenario, the effect of changing the number of users $M$ in the downlink NOMA system on the performance of the system in the perfect CSI case, in terms of energy efficiency and outage probability, is investigated. For various values of $M$ between 1 and 10 and under the same circumstances in the previous experiments with the same threshold SINR=1 (minimum data rate is $1 \mathrm{bps}$ ), the outage probability of the cell-edge user and the energy efficiency of the system are evaluated and presented in Figure 5 and Figure 6 , respectively. The outage probability decreases when the transmission power increases for all values of $M$. It is obvious from Figure 5 that when $M=1$, the outage probability is zero when the transmission power is adequate to achieve the threshold SINR level. However, the outage probability of the cell-edge user increases when the number of 
users in the cell increases at each transmitted power value. That is because the interference from other users increased and the allocated power to the cell-edge user is inadequate to attain the required SINR. On the other hand, the energy efficiency increases when $M$ increases until maximum value and then it decrease again for higher number of users. This is because that according to the propose power allocation algorithm, if the SINR at the celledge user is lower that the threshold level, the system resources will be re-allocated to $M-1$ users. Although at higher values of transmitted power, the outage probability is not zero because of the required level of the SINR.

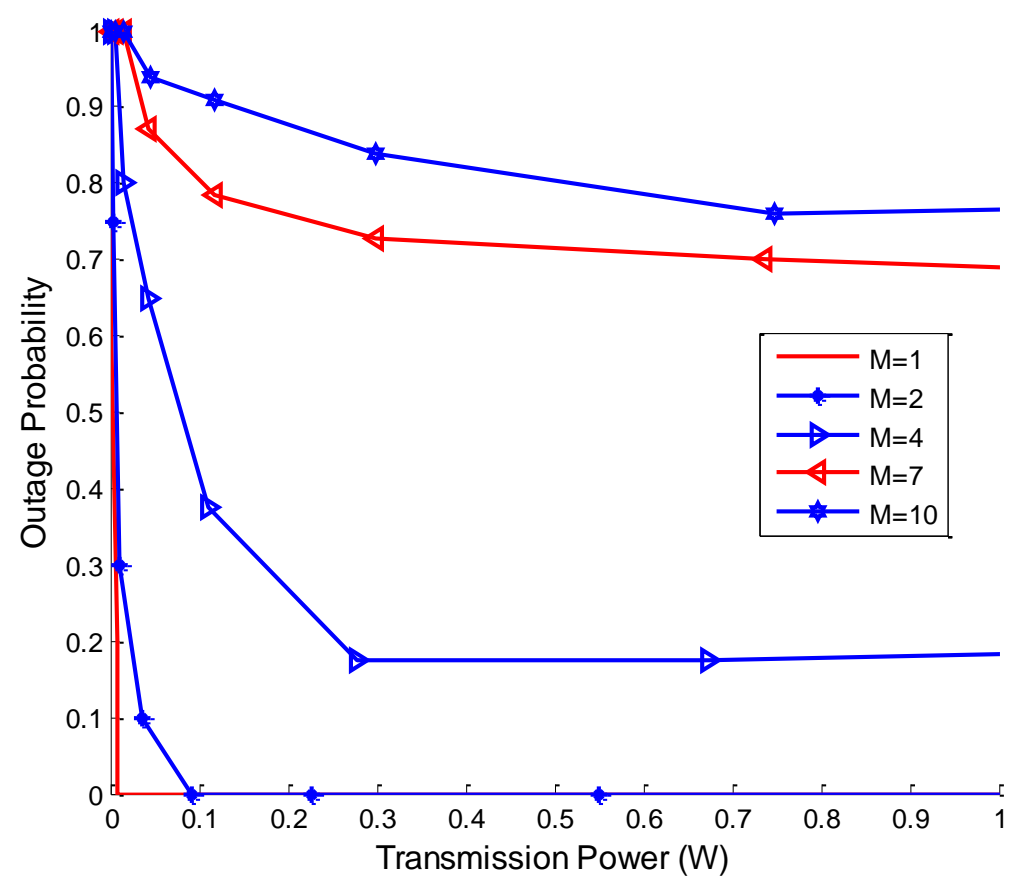

Figure 5. Outage Probability versus transmission power at various number of users values

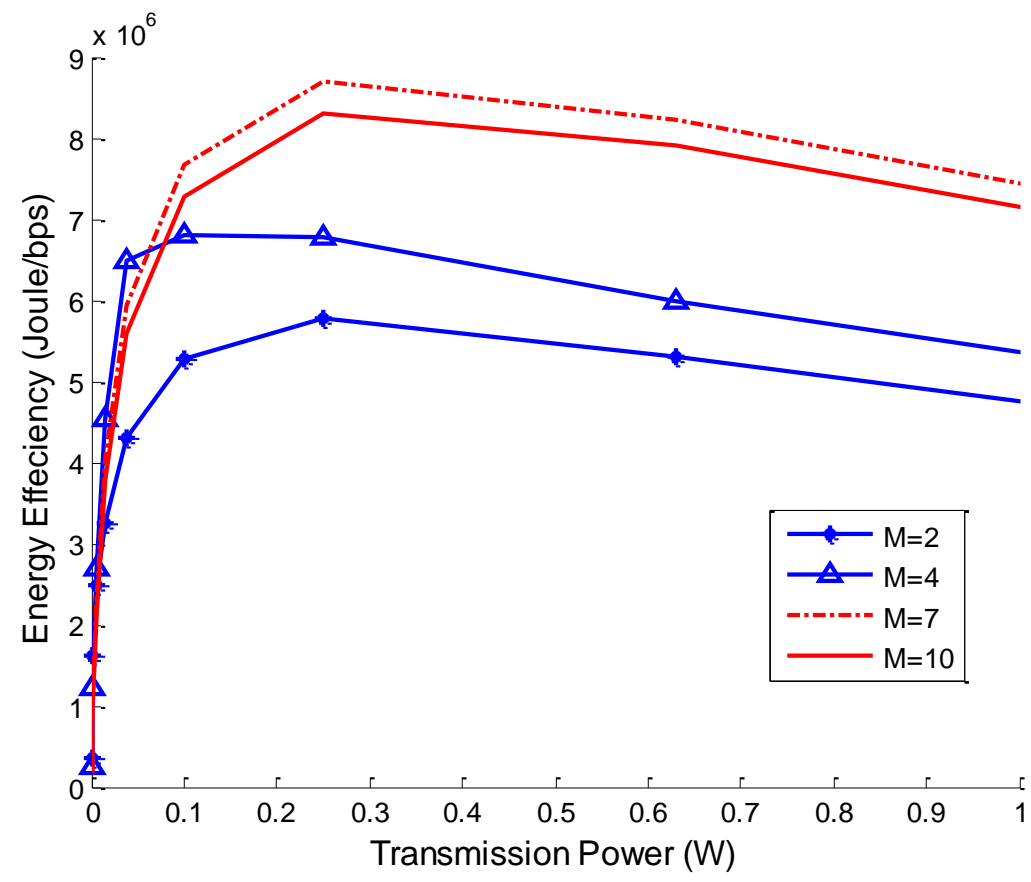

Figure 6. Energy efficiency versus transmission power at various number of users values 


\section{Conclusions}

Obtaining full channel state information at the BS in the downlink cellular system is practically difficult. In this paper, a low complexity PA algorithm for downlink NOMA system is proposed. The allocated power to any user in a cellular cell is inversely proportional to its channel strength to guarantee that the desired signal could be extracted by the SIC technique at the receiver's terminal. The proposed algorithm's performance is investigated in terms of energy efficiency and outage probability at different levels of transmission power and different estimation error values. Results prove that the proposed algorithm outperforms the conventional OMA in perfect CSI and imperfect CSI cases. A future direction of our work is to integrate the proposed algorithm in a multiple-input multiple-output (MIMO) environment and heterogeneous NOMA system and evaluate the performance for the cell edge user in terms of energy efficiency and spectrum efficiency.

\section{References}

[1] J.G. Andrews, S. Buzzi, W. Choi, S.V. Hanly, A. Lozano, A.C.K. Soong, and J.C. Zhang, "What will 5G be?," IEEE Journal on Selected Areas in Communications, Vol. 32, No. 6, pp. 1065-1082, 2014.

[2] M. Agiwal, A. Roy, and N. Saxena, "Next generation 5G wireless networks: a comprehensive survey," IEEE Communications Surveys \& Tutorials, Vol. 18, No. 3, pp. 1617-1655, 2016.

[3] W. Bai, T. Yao, H. Zhang, and V. C. M. Leung, "Research on channel power allocation of fog wireless access network based on NOMA," IEEE Access, Vol. 7, pp. 32867-32873, 2019.

[4] Z. Zhang Y. Xiao, Z. Ma, M. Xiao, Z. Ding, X. Lei, G.K. Karagiannidis, and P. Fan, "6G wireless networks: vision, requirements, architecture, and key technologies," IEEE Vehicular Technology Magazine, Vol. 14, No. 3, pp. 28-41, 2019.

[5] Y. Wang, B. Ren, S. Sun, S. Kang, and X. Yue, "Analysis of non-orthogonal multiple access for 5G," China Communications, Vol. 13, pp. 52-66, 2016.

[6] S.M.R. Islam, N. Avazov, O.A. Dobre, and K. Kwak, "Power-domain non-orthogonal multiple access (NOMA) in 5G systems: potentials and challenges," IEEE Communications Surveys \& Tutorials, Vol. 19, No. 2, pp. 721-742, 2017.

[7] Z. Yang, J.A. Hussein, P. Xu, Z. Ding, and Y. Wu, "Power allocation study for nonorthogonal multiple access networks with multicast-unicast transmission," IEEE Transactions on Wireless Communications, Vol. 17, No. 6, pp. 3588-3599, 2018.

[8] R. Aldebes, K. Dimyati, and E. Hanafi, "Game-theoretic power allocation algorithm for downlink NOMA cellular system," Electronics Letters, Vol. 55, No. 25, pp. 1361$1364,2019$.

[9] X. Song, L. Dong, J. Wang, L. Qin, and X. Han, "Energy-efficient power allocation for downlink NOMA heterogeneous networks with imperfect CSI," IEEE Access, Vol. 7, pp. 39329-39340, 2019.

[10] Y. Zhang, H. Wang, T. Zheng, and Q. Yang, "Energy-efficient transmission design in non-orthogonal multiple access," IEEE Transactions on Vehicular Technology, Vol. 66, No. 3, pp. 2852-2857, 2017.

[11] J. Shi, W. Yu, Q. Ni, W. Liang, Z. Li, and P. Xiao, "Energy-efficient resource allocation in hybrid non-orthogonal multiple access systems," IEEE Transactions on Communications, Vol. 67, No. 5, pp. 3496-3511, 2019.

[12] H. Zhang, B. Wang, C. Jiang, K. Long, A. Nallanathan, V.C. M. Leung, and H.V. Poor, "Energy-efficient dynamic resource optimization in NOMA system," IEEE Transactions on Wireless Communications, Vol. 17, No. 9, pp. 5671-5683, 2018. 
[13] Q. Vien, T.A. Le, B. Barn, and C.V. Phan, "Optimising energy efficiency of nonorthogonal multiple access for wireless backhaul in heterogeneous cloud radio access network," IET Communications, Vol. 10, No. 18, pp. 2516-2524, 2016.

[14] N. Glei and R.B. Chibani, "Power allocation for energy-efficient downlink NOMA systems," In: 19th International Conference on Sciences and Techniques of Automatic Control and Computer Engineering (STA), Sousse, Tunisia, pp. 611-613, 2019.

[15] X. Chen, R. Jia, and D.W.K. Ng, "On the design of massive non-orthogonal multiple access with imperfect successive interference cancellation," IEEE Transactions on Communications, Vol. 67, No. 3, pp. 2539-2551, 2019.

[16] S. Arzykulov, T.A. Tsiftsis, G. Nauryzbayev, and M. Abdallah, "Outage performance of cooperative underlay CR-NOMA with imperfect CSI," IEEE Communications Letters, Vol. 23, No. 1, pp. 176-179, 2019.

[17] M.R. Zamani, M. Eslami, M. Khorramizadeh, and Z. Ding, "Energy-efficient power allocation for NOMA with imperfect CSI," IEEE Transactions on Vehicular Technology, Vol. 68, No. 1, pp. 1009-1013, 2019.

[18] S. Timotheou and I. Krikidis, "Fairness for non-orthogonal multiple access in 5G systems," IEEE Signal Processing Letters, Vol. 22, No. 10, pp. 1647-1651, 2015.

[19] M.S. Ali, H. Tabassum, and E. Hossain, "Dynamic user clustering and power allocation for uplink and downlink non-orthogonal multiple access (NOMA) systems," IEEE Access, Vol. 4, pp. 6325-6343, 2016.

[20] K.S. Ali, M. Haenggi, H. ElSawy, A. Chaaban, and M. Alouini, "Downlink nonorthogonal multiple access (NOMA) in poisson networks," IEEE Transactions on Communications, Vol. 67, No. 2, pp. 1613-1628, 2019.

[21] F. Mokhtar, M.R. Mili, F. Eslami, F. Ashtiani, B. Makki, M. Mirmohseni, M. NasiriKenari, and T. Svensson, "Download elastic traffic rate optimization via NOMA protocols," IEEE Transactions on Vehicular Technology, Vol. 68, No. 1, pp. 713-727, 2019.

[22] M.S. Ali, E. Hossain, A. Al-Dweik, and D.I. Kim, "Downlink power allocation for CoMP-NOMA in multi-cell networks," IEEE Transactions on Communications, Vol. 66, No. 9, pp. 3982-3998, 2018.

[23] Z. Wei, L. Yang, D. W.K. Ng, J. Yuan, and L. Hanzo, "On the performance gain of NOMA over OMA in uplink communication systems," IEEE Transactions on Communications, Vol. 68, No. 1, pp. 536-568, 2020.

[24] A.S. Marcano and H.L. Christiansen, "Impact of NOMA on network capacity dimensioning for 5G HetNets, ” IEEE Access, Vol. 6, pp. 13587-13603, 2018. 